Article

\title{
Catalytic Enantioselective Aryl Transfer to Aldehydes Using Chiral 2,2'-Bispyrrolidine-Based Salan Ligands
}

\section{Xuefeng Jia ${ }^{1,2}$, Aijun Lin ${ }^{1}$, Zhijie Mao ${ }^{1}$, Chengjian Zhu ${ }^{1,3, *}$ and Yixiang Cheng ${ }^{4, *}$}

1 School of Chemistry and Chemical Engineering, State Key Laboratory of Coordination Chemistry, Nanjing University, Nanjing, 210093, China

2 School of Chemistry and Materials Science, Shanxi Normal University, Linfen, 041004, China

3 State Key Laboratory of Organometallic Chemistry, Shanghai Institute of Organic Chemistry, Chinese Academy of Sciences, Shanghai, 200032, China

4 School of Chemistry and Chemical Engineering, Key Laboratory of Mesoscopic Chemistry of MOE, Nanjing University, Nanjing, 210093, China

* Authors to whom correspondence should be addressed; E-Mails: cjzhu@nju.edu.cn (C.Z.); yxcheng@nju.edu.cn (Y.C.); Tel.: (+86)25-83594886; Fax: (+86)25-83594886.

Received: 24 December 2010; in revised form: 15 March 2011 / Accepted: 16 March 2011 / Published: 6 April 2011

\begin{abstract}
Chiral $C_{2}$-symmetric diamines have emerged as versatile auxiliaries or ligands in numerous asymmetric transformations. Chiral 2,2'-bispyrrolidine-based salan ligands were prepared and applied to the asymmetric aryl transfer to aldehydes with arylboronic acids as the source of transferable aryl groups. The corresponding diarylmethanols were obtained in high yields with moderate to good enantioselectivitives of up to $83 \%$ ee.
\end{abstract}

Keywords: 2,2'-bispyrrolidine; salan ligands; aryl transfer; arylboronic acid; enantioselectivity

\section{Introduction}

Chiral diarylmethanols are important intermediates and precursors for the synthesis of pharmacologically and biologically active compounds [1-8]. Therefore, the development of effective catalyst systems for the synthesis of these compounds is of significant importance for organic chemists. The scientifically important protocols for the synthesis of chiral diarylmethanols commonly 
involve two strategies: (1) the asymmetric reduction of prochiral diaryl ketones [9-13], (2) the enantioselective aryl transfer to aromatic aldehydes [14-16]. The reduction method requires an ortho substituent on one of the aryls or electronic different aryl groups for optimum results. The second method seems easy to realize chiral induction due to the large steric and electronic differences between an aryl group and a hydrogen atom on the aldehyde substrates with diphenylzinc. As reported previously, many functionalized diarylzincs used as the transferring nucleophiles are unstable and difficult to synthesize, so the method of the aryl transfer to aldehyde is greatly limited. Recently, an elegant method that the arylzinc species prepared in situ by transmetalation between organoboron [17-19] or organoboronic derivatives [20-26] and diethylzinc has been proposed as an alternative for the synthesis of salt-free organozinc reagents. We have also successfully developed an efficient and practical method for the synthesis of diarylmethanols by transmetalation using the arylboronic acid in the presence of trimethylgallium [27]. These methods allow the exploitation of a broad range of substituted aryl transfer reagents since numerous arylboronic acids are commercially available, and a lot of excellent ligands were developed and applied to the asymmetric aryl transfer reaction with good results [28-41]. For the future, the introduction of the new, effective and more easily available catalysts is also a field of continuous interest for the catalytic aryl transfer reaction.

Chiral $C_{2}$-symmetric diamines have emerged as versatile auxiliaries or ligands in numerous asymmetric transformations [42-44]. $(R, R)-2,2^{\prime}$ '-bispyrrolidine, initially developed by Hirama, was synthesized by various routes [45-50], and its derivatives had been successfully employed as chiral ligands or organocatalysts in many asymmetric reactions [51-59]. So far, the application of 2,2'bispyrrolidine-based salan ligands $[60,61]$ in asymmetric catalysis has not been reported. We describe herein our efforts toward the synthesis of optically active diarylmethanols through the asymmetric aryl transfer to aldehydes under the catalysis of $(R, R)-2,2$ '-bispyrrolidine-based salan ligands.

Figure 1. Structures of Ligands L1-L6.

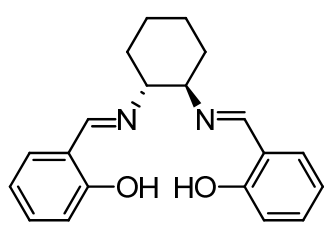

L1

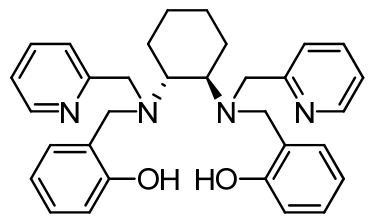

L4

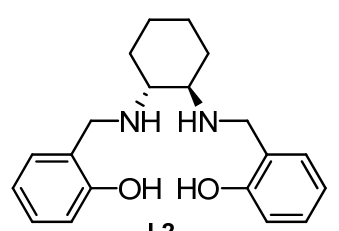

L2

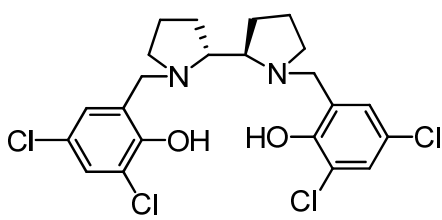

L5

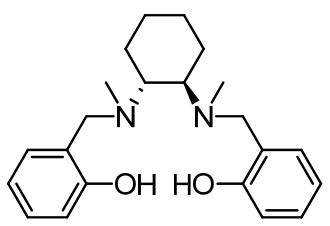

L3

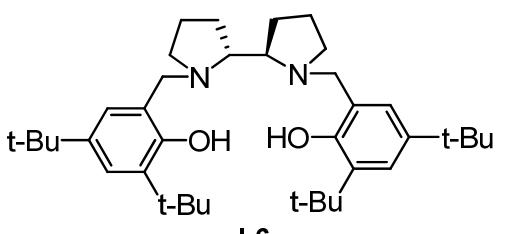

\section{Results and Discussion}

A preliminary study was performed to test the catalytic property of the ligands L1-L6 (Figure 1) in the asymmetric phenyl transfer reaction to 4 -nitrobenzaldehyde at $0{ }^{\circ} \mathrm{C}$. As is evident from Table 1 , the resulting products could be obtained in moderate yield, but low enantioselectivity when $(1 R, 2 R)$ cyclohexane-1,2-diamine-based ligands L1-L4 were tested (Table 1, entries 1-4). Gratifyingly, we found that the ligands $\mathbf{L} 5$ and $\mathbf{L 6}$ were more effective in this reaction (Table 1, entries 5-6). The ee 
value of the product could be increased to $63 \%$ when the reaction was carried out at $-25{ }^{\circ} \mathrm{C}$ (Table 1 , entry 7). Increasing catalyst loading had a positive impact on both the yield and enantioselectivity. The best result was obtained in $88 \%$ yield with $83 \%$ ee while using 20 mol\% of L6 (Table 1, entry 9).

Table 1. Asymmetric Phenyl Transfer to 4-nitrobenzaldehyde. ${ }^{a}$

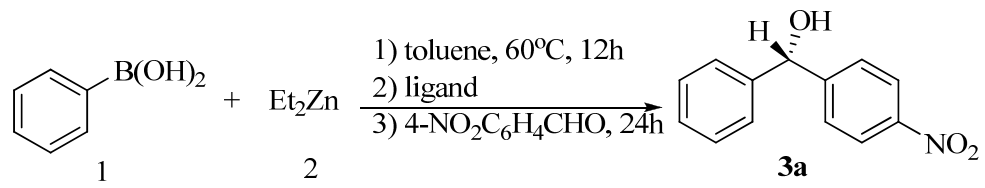

\begin{tabular}{llllll}
\hline Entry & Ligand & Mol\% & $\mathbf{T}\left({ }^{\circ} \mathbf{C}\right)$ & Yield(\%) $^{\mathbf{b}}$ & Ee(\%) \\
\hline 1 & L1 & 10 & 0 & 66 & 6 \\
2 & L2 & 10 & 0 & 73 & 11 \\
3 & L3 & 10 & 0 & 69 & 16 \\
4 & L4 & 10 & 0 & 80 & 3 \\
5 & L5 & 10 & 0 & 74 & 31 \\
6 & L6 & 10 & 0 & 84 & 43 \\
7 & L6 & 10 & -25 & 70 & 63 \\
8 & L6 & 15 & -25 & 80 & 71 \\
9 & L6 & 20 & -25 & 88 & $83(S)^{d}$ \\
\hline
\end{tabular}

${ }^{a}$ All the reactions were carried out on $0.2 \mathrm{mmol}$ scale of substrates with 2 equiv of arylboronic acid and 6 equiv of $\mathrm{Et}_{2} \mathrm{Zn}$ in toluene for $24 \mathrm{~h} .{ }^{b}$ Isolated yields. ${ }^{c}$ Determined by HPLC with a Chiralcel OB-H column. ${ }^{d}$ The absolute configuration of the products were determined by comparison with literature values.

After having established the optimal protocol for the asymmetric phenyl transfer reaction, we further extended the reaction to a series of aldehyde substrates (Table 2). The electronic properties of the aromatic rings of the aldehydes have a significant influence on the enantioselectivity in this reaction. The aldehydes with electron-withdrawing substituents provided better results than those with electron-donating substituents in terms of ee values. 4-Nitrobenzaldehyde gave the corresponding diarylmethanol with $83 \%$ ee, but 4-methoxybenzaldehyde only with $11 \%$ ee (Table 2, entries 1, 2 and 10). Similar results were obtained when 3-substituted-benzaldehydes (Table 2, entries 3 and 9) or 2substituted-benzaldehydes (Table 2, entries 5,6 and 8) were tested. However, an exception was observed for 2-nitrobenzaldehyde (Table 2, entry 4), presumably caused by the chelating effect of the $\mathrm{NO}_{2}$ group with the lewis acids $[62,63]$. The enantioselectivity was also found to be influenced by the steric effect with the same exception of 2-nitrobenzaldehyde. ortho-Substituted (-Cl or -Me) benzaldehydes gave higher ee values (Table 2, entries 6 vs 2 or 8 vs 9). It should be noted that the reaction of 2-naphthaldehyde proceeded well, giving 70\% ee and good yield (Table 2, entry 11), and $\alpha, \beta$-unsaturated cinnamaldehyde gave the corresponding product with only moderate enantioselectivity (Table 2, entry 12).

We also further investigated the asymmetric aryl transfer to aromatic aldehydes with substituted phenylboronic acids. As shown in Table 3, when 4-chlorophenylboronic acid was chosen as the aryl source and 4-nitrobenzaldehyde as the substrate, $71 \%$ ee was obtained (Table 3, entry 1). And 54\% ee was obtained when 4-methoxylphenylboronic acid was tested (Table 3, entry 3). 
Table 2. Asymmetric Phenyl Transfer to Aromatic Aldehydes. ${ }^{a}$

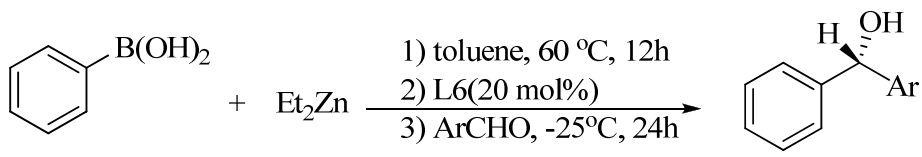

1

2

\begin{tabular}{|c|c|}
\hline \multicolumn{2}{|c|}{ 3a-1 } \\
\hline Yield $(\%)^{b}$ & $\operatorname{Ee}(\%)^{c, d}$ \\
\hline 88 & $83(S)$ \\
\hline 80 & $41(S)$ \\
\hline 91 & $75(S)$ \\
\hline 85 & $41(R)$ \\
\hline 72 & $80(R)$ \\
\hline 84 & $73(S)$ \\
\hline 85 & $26(S)$ \\
\hline 76 & $60(S)$ \\
\hline 78 & $52(S)$ \\
\hline 80 & $11(S)$ \\
\hline 80 & $70(S)$ \\
\hline 76 & $47(R)$ \\
\hline
\end{tabular}

Table 3. Asymmetric Aryl Transfer to Aldehydes. ${ }^{a}$

$$
\begin{aligned}
& \text { 1) toluene, } 60^{\circ} \mathrm{C}, 12 \mathrm{~h}
\end{aligned}
$$

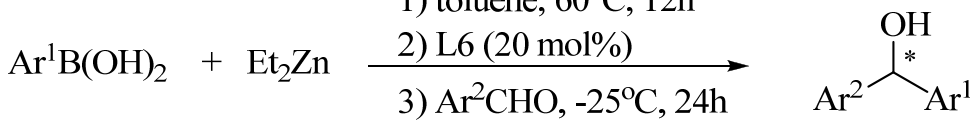

\begin{tabular}{llllll}
\hline Entry & $\mathbf{A r}$ & $\mathbf{A r}^{\mathbf{1}}$ & Product & Yield (\%) & $\mathbf{E e ~ ( \% ) ~}^{\boldsymbol{c}}$ \\
\hline 1 & $4-\mathrm{Cl} \mathrm{C}_{6} \mathrm{H}_{4}$ & $4-\mathrm{NO}_{2} \mathrm{C}_{6} \mathrm{H}_{4}$ & $4 \mathrm{a}$ & 75 & 71 \\
2 & $4-\mathrm{Cl} \mathrm{C}_{6} \mathrm{H}_{4}$ & $3-\mathrm{BrC}_{6} \mathrm{H}_{4}$ & $4 \mathrm{~b}$ & 82 & 55 \\
3 & $4-\mathrm{MeOC}_{6} \mathrm{H}_{4}$ & $4-\mathrm{NO}_{2} \mathrm{C}_{6} \mathrm{H}_{4}$ & $4 \mathrm{c}$ & 78 & $54(S)^{d}$ \\
4 & $4-\mathrm{MeOC}_{6} \mathrm{H}_{4}$ & $3-\mathrm{NO}_{2} \mathrm{C}_{6} \mathrm{H}_{4}$ & $4 \mathrm{~d}$ & 70 & 24 \\
5 & $3,5-\mathrm{diMeC}_{6} \mathrm{H}_{3}$ & $\mathrm{C}_{6} \mathrm{H}_{5}$ & $4 \mathrm{e}$ & 70 & 48 \\
\hline
\end{tabular}

${ }^{a}$ All reactions were carried out on $0.15 \mathrm{mmol}$ scale of substrates with 2 equiv of arylboronic acid and 6 equiv of $\mathrm{Et}_{2} \mathrm{Zn}$ in toluene at $-25{ }^{\circ} \mathrm{C}$ for $24 \mathrm{~h}$ in the presence of $20 \mathrm{~mol} \%$ ligand; ${ }^{b}$ Isolated yields; ${ }^{c}$ Enantiomeric excess was determined by HPLC with a Chiralcel OB-H, OD-H or AD-H column; ${ }^{d}$ The absolute configuration of the products were determined by comparison with literature values.

\section{Experimental}

\subsection{General}

All reactions were carried out under an argon atmosphere using standard Schlenk techniques. Solvents were dried and distilled prior to use according to standard methods. Unless otherwise indicated, all materials were obtained from commercial sources and liquid aldehydes were freshly distilled before use. For thin-layer chromatography (TLC), compounds were visualized by irradiation with UV light on GF 254 silica gel plates. ${ }^{1} \mathrm{H}-\mathrm{NMR}$ and ${ }^{13} \mathrm{C}$-NMR spectra were recorded in $\mathrm{CDCl}_{3}$ on a Bruker ARX-300 spectrometer with chemical shifts being referenced to $\mathrm{SiMe}_{4}$ used as internal 
standard. The coupling constants $J$ are given in Hz. HPLC analysis were performed on a chiral column (Daicel Chiralcel OB-H, OD-H or AD-H column) on a Chromatography Interface 600 Series Link instrument and Series 200 pump), with Series 200 UV/VIS detection at $254 \mathrm{~nm}$. The solvent system used has hexane (A)-2-propanol (B) in the indicated proportions. Optical rotations were measured on Rudolph Research Analytical Autopol III Automatic Polarimeter equipped with a $100 \mathrm{~mm}$ cell. Mass spectra (EI-MS) were taken using a Shimadzu GCMS-QP2010 mass spectrometer. High Resolution Mass Spectra (HRMS) were taken using a LTQ Orbitrap XL ThermoFisher unit.

\subsection{Typical Procedure for the Asymmetric Aryl Transfer Reaction}

In a $20 \mathrm{~mL}$ flame-dried Schlenk reaction tube, diethylzinc $(0.9 \mathrm{mmol}, 6$ equiv, $1.5 \mathrm{M}$ in toluene solution) was added dropwise to a solution of phenylboronic acid ( $0.3 \mathrm{mmol}, 2$ equiv) in toluene (3 mL) under an argon atmosphere. After stirring for $12 \mathrm{~h}$ at $60^{\circ} \mathrm{C}$, a toluene solution of $\mathbf{L 6}$ (20 mol\%) was introduced. The reaction was stirred for an additional 30 minutes and cooled to $-25{ }^{\circ} \mathrm{C}$ followed by the addition of aldehydes $(0.15 \mathrm{mmol})$. After completion of the reaction (monitored by TLC), the reaction solution was quenched with saturated aqueous $\mathrm{NH}_{4} \mathrm{Cl}(3 \mathrm{~mL})$ and further extracted with ethyl acetate $(3 \times 5 \mathrm{~mL})$. The combined organic layer was dried over $\mathrm{Na}_{2} \mathrm{SO}_{4}$. Evaporation of the solvent gave the crude product, which was further purified by preparative TLC to afford the corresponding chiral diarylmethanols.

(S)-4-Nitrophenyl(phenyl)methanol (3a). ${ }^{1} \mathrm{H}-\mathrm{NMR}: \delta 8.19(\mathrm{~d}, J=7.2 \mathrm{~Hz}, 2 \mathrm{H}), 7.58(\mathrm{~d}, J=7.2 \mathrm{~Hz}$, 2H), 7.37-7.33 (m, 5H), $5.92(\mathrm{~s}, 1 \mathrm{H}), 2.25$ (brs, 1H). 83\% ee determined by HPLC with a Chiralcel OB-H column $(\mathrm{A} / \mathrm{B}=70: 30,0.8 \mathrm{~mL} / \mathrm{min}$, $u v 230 \mathrm{~nm}): \mathrm{t}_{\mathrm{R}}=21.05 \mathrm{~min}$ (minor), $\mathrm{t}_{\mathrm{R}}=35.74$ min (major). $[\alpha]_{\mathrm{D}}^{23}=+31.6(\mathrm{c}=0.50, \mathrm{EtOH})$.

(S)-4-Chlorophenyl(phenyl)methanol (3b). ${ }^{1} \mathrm{H}-\mathrm{NMR}$ : $\delta$ 7.38-7.33 (m, 4H), 7.31-7.27 (m, 5H), 5.80 (s, 1H), 2.20 (brs, 1H). 41\% ee determined by HPLC with a Chiralcel OB-H column (A/B = 90:10, $1.0 \mathrm{~mL} / \mathrm{min}$, uv $230 \mathrm{~nm}): \mathrm{t}_{\mathrm{R}}=10.21 \mathrm{~min}$ (minor), $\mathrm{t}_{\mathrm{R}}=18.33 \mathrm{~min}$ (major). $[\alpha]_{\mathrm{D}}{ }^{23}=+5.9(c=0.64$, $\mathrm{EtOH})$.

(S)-3-Nitrophenyl(phenyl)methanol (3c). ${ }^{1} \mathrm{H}-\mathrm{NMR}: \delta 8.30(\mathrm{~s}, 1 \mathrm{H}), 8.11(\mathrm{~d}, J=8.1 \mathrm{~Hz}, 1 \mathrm{H}), 7.72(\mathrm{~d}$, $J=7.8 \mathrm{~Hz}, 1 \mathrm{H}), 7.50(\mathrm{t}, J=7.8 \mathrm{~Hz}, 1 \mathrm{H}), 7.40-7.29(\mathrm{~m}, 5 \mathrm{H}), 5.92$ (s, 1H), 2.13 (brs, 1H). 75\% ee determined by HPLC with a Chiralcel OB-H column $(\mathrm{A} / \mathrm{B}=80: 20,0.8 \mathrm{~mL} / \mathrm{min}$, uv $230 \mathrm{~nm})$ : $\mathrm{t}_{\mathrm{R}}=34.19 \min$ (minor), $\mathrm{t}_{\mathrm{R}}=47.40 \min$ (major). $[\alpha]_{\mathrm{D}}^{23}=+42.5(c=0.40, \mathrm{EtOH})$.

(R)-2-Nitrophenyl(phenyl)methanol (3d). ${ }^{1} \mathrm{H}-\mathrm{NMR}: \delta 7.94$ (dd, $\left.J=7.8,1.5 \mathrm{~Hz}, 1 \mathrm{H}\right), 7.75$ (dd, $J=7.8$, $1.5 \mathrm{~Hz}, 1 \mathrm{H}), 7.64$ (dt, $J=7.5,1.2 \mathrm{~Hz}, 1 \mathrm{H}), 7.46$ (t, $J=7.8,1.5 \mathrm{~Hz}, 1 \mathrm{H}), 7.36-7.29(\mathrm{~m}, 5 \mathrm{H}), 6.44$ (s, 1H), 2.02 (brs, 1H). 41\% ee determined by HPLC with a Chiralpark AD-H column (A/B = 90:10, $0.8 \mathrm{~mL} / \mathrm{min}$, uv $254 \mathrm{~nm}): \mathrm{t}_{\mathrm{R}}=13.57 \mathrm{~min}($ major$), \mathrm{t}_{\mathrm{R}}=14.62 \min ($ minor $) ;[\alpha]_{\mathrm{D}}^{23}=11.2(c=0.32$, $\mathrm{EtOH})$. 
(R)-2-Trifluoromethylphenyl(phenyl)methanol (3e). ${ }^{1} \mathrm{H}-\mathrm{NMR}: \delta 7.66(\mathrm{t}, J=7.8 \mathrm{~Hz}, 2 \mathrm{H}), 7.55(\mathrm{t}$, $J=7.8 \mathrm{~Hz}, 1 \mathrm{H}), 7.42-7.32(\mathrm{~m}, 5 \mathrm{H}), 7.30-7.27(\mathrm{~m}, 1 \mathrm{H}), 6.32(\mathrm{~s}, 1 \mathrm{H}), 1.99$ (brs, $1 \mathrm{H}) .80 \%$ ee determined by HPLC with a Chiralcel OD-H column $(\mathrm{A} / \mathrm{B}=90: 10,0.5 \mathrm{~mL} / \mathrm{min}$, uv $254 \mathrm{~nm})$ : $\mathrm{t}_{\mathrm{R}}=9.33 \min ($ major $), \mathrm{t}_{\mathrm{R}}=11.79 \min ($ minor $) .[\alpha]_{\mathrm{D}}{ }^{23}=-37.2(c=0.5, \mathrm{EtOH})$.

(S)-2-Chlorophenyl(phenyl)methanol (3f). ${ }^{1} \mathrm{H}-\mathrm{NMR}: \delta 7.60(\mathrm{~d}, J=7.8 \mathrm{~Hz}, 1 \mathrm{H}), 7.42-7.39(\mathrm{~m}, 2 \mathrm{H})$, 7.36-7.28 (m, 5H), 7.25-7.22 (m, 1H), 6.24 (s, 1H), 2.05 (brs, 1H). 73\% ee determined by HPLC with a Chiralcel OB-H column $(\mathrm{A} / \mathrm{B}=90: 10,1.0 \mathrm{~mL} / \mathrm{min}$, uv $230 \mathrm{~nm}): \mathrm{t}_{\mathrm{R}}=8.97 \mathrm{~min}$ (minor), $\mathrm{t}_{\mathrm{R}}=10.00 \min$ (major). $[\alpha]_{\mathrm{D}}^{23}=-20.6(c=0.64, \mathrm{EtOH})$.

(S)-3-Bromophenyl(phenyl)methanol (3g). ${ }^{1} \mathrm{H}-\mathrm{NMR}: \delta 7.57$ (s, 1H), 7.42-7.35 (m, 5H), 7.33-7.27 (m, $2 \mathrm{H}), 7.20(\mathrm{t}, J=7.8 \mathrm{~Hz}, 1 \mathrm{H}), 5.78(\mathrm{~s}, 1 \mathrm{H}), 2.33$ (brs, $1 \mathrm{H}) .26 \%$ ee determined by HPLC with a Chiralcel OB-H column $(\mathrm{A} / \mathrm{B}=90: 10,1.0 \mathrm{~mL} / \mathrm{min}$, uv $230 \mathrm{~nm}): \mathrm{t}_{\mathrm{R}}=15.17 \mathrm{~min}$ (minor), $\mathrm{t}_{\mathrm{R}}=27.81 \min$ (major). $[\alpha]_{\mathrm{D}}^{23}=+11.4(c=0.76, \mathrm{EtOH})$.

(S)-2-Methylphenyl(phenyl)methanol (3h). ${ }^{1} \mathrm{H}-\mathrm{NMR}: \delta 7.53(\mathrm{~d}, J=9.0 \mathrm{~Hz}, 1 \mathrm{H}), 7.34-7.21(\mathrm{~m}, 7 \mathrm{H})$, $7.16(\mathrm{t}, J=8.1 \mathrm{~Hz}, 1 \mathrm{H}), 6.02(\mathrm{~s}, 1 \mathrm{H}), 2.26(\mathrm{~s}, 3 \mathrm{H}), 1.95(\mathrm{~s}, 1 \mathrm{H}) .60 \%$ ee determined by HPLC with a Chiralcel OB-H column (A/B = 90:10, $1.0 \mathrm{~mL} / \mathrm{min}$, uv $230 \mathrm{~nm}): \mathrm{t}_{\mathrm{R}}=9.47 \mathrm{~min}($ minor $), \mathrm{t}_{\mathrm{R}}=10.6 \mathrm{~min}$ (major). $[\alpha]_{\mathrm{D}}^{23}=-19.3(c=0.30, \mathrm{EtOH})$.

(S)-3-Methylphenyl(phenyl)methanol (3i). ${ }^{1} \mathrm{H}-\mathrm{NMR}: \delta 7.41-7.34(\mathrm{~m}, 4 \mathrm{H}), 7.30-7.27$ (m, 2H), 7.24-7.16 (m, 2H), 7.09 (d, $J=7.5 \mathrm{~Hz}, 1 \mathrm{H}), 5.81(\mathrm{~s}, 1 \mathrm{H}), 2.35$ (s, 3H), 2.02 (brs, 1H). 52\% ee determined by HPLC with a Chiralcel OB-H column $(\mathrm{A} / \mathrm{B}=90: 10,1.0 \mathrm{~mL} / \mathrm{min}$, uv $230 \mathrm{~nm})$ : $\mathrm{t}_{\mathrm{R}}=12.39 \min ($ minor $), \mathrm{t}_{\mathrm{R}}=21.34 \min ($ major $) .[\alpha]_{\mathrm{D}}^{23}=-15.8(c=0.34, \mathrm{EtOH})$.

(S)-4-Methoxylphenyl(phenyl)methanol (3j). ${ }^{1} \mathrm{H}-\mathrm{NMR}$ : $\delta$ 7.37-7.34 (m, 3H), 7.30-7.26 (m, 4H), 6.88 $(\mathrm{d}, J=9.0 \mathrm{~Hz}, 2 \mathrm{H}), 5.81(\mathrm{~s}, 1 \mathrm{H}), 3.78(\mathrm{~s}, 3 \mathrm{H}), 2.23(\mathrm{brs}, 1 \mathrm{H}) .11 \%$ ee determined by HPLC with a Chiralcel OB-H column $(\mathrm{A} / \mathrm{B}=90: 10,1.0 \mathrm{~mL} / \mathrm{min}$, uv $230 \mathrm{~nm}): \mathrm{t}_{\mathrm{R}}=21.79 \mathrm{~min}$ (minor), $\mathrm{t}_{\mathrm{R}}=24.03 \min$ (major). $[\alpha]_{\mathrm{D}}^{23}=8.1(c=0.42, \mathrm{EtOH})$.

(S)-2-Naphathyl(phenyl)methanol (3k). ${ }^{1} \mathrm{H}-\mathrm{NMR}: \delta 7.90(\mathrm{~s}, 1 \mathrm{H}), 7.86-7.79(\mathrm{~m}, 3 \mathrm{H}), 7.50-7.42$ (m, 5H), 7.38-7.28 (m, 3H), $6.01(\mathrm{~s}, 1 \mathrm{H}), 2.06$ (brs, 1H). 70\% ee determined by HPLC with a Chiralcel OD-H column $(\mathrm{A} / \mathrm{B}=85: 15,0.8 \mathrm{~mL} / \mathrm{min}$, uv $230 \mathrm{~nm}): \mathrm{t}_{\mathrm{R}}=12.71 \mathrm{~min}$ (major), $\mathrm{t}_{\mathrm{R}}=15.08 \mathrm{~min}$ (minor). $[\alpha]_{\mathrm{D}}^{23}=-18.4(\mathrm{c}=0.46, \mathrm{EtOH})$.

(R)-1, 3-Diphenylprop-2-en-1-ol (3l). ${ }^{1} \mathrm{H}-\mathrm{NMR}: \delta$ 7.47-7.33 (m, 5H), 7.32-7.27 (m, 3H), 7.29-7.24 $(\mathrm{m}, 2 \mathrm{H}), 6.70(\mathrm{~d}, J=15.6 \mathrm{~Hz}, 1 \mathrm{H}), 6.40(\mathrm{dd}, J=6.3,15.6 \mathrm{~Hz}, 1 \mathrm{H}), 5.40(\mathrm{~d}, J=6.6 \mathrm{~Hz}, 1 \mathrm{H}), 2.15$ (brs, $1 \mathrm{H}) .47 \%$ ee determined by HPLC with a Chiralcel OD-H column $(\mathrm{A} / \mathrm{B}=80: 20,0.8 \mathrm{~mL} / \mathrm{min}$, uv $254 \mathrm{~nm}): t_{\mathrm{R}}=9.31 \min ($ minor $), \mathrm{t}_{\mathrm{R}}=11.14 \min$ (major). $[\alpha]_{\mathrm{D}}{ }^{20}=+13.5(c=0.40, \mathrm{EtOH})$.

4-Chlorophenyl(4-nitrophenyl)methanol (4a). ${ }^{1} \mathrm{H}-\mathrm{NMR}: \delta 8.20(\mathrm{~d}, J=8.7 \mathrm{~Hz}, 2 \mathrm{H}), 7.55(\mathrm{~d}, J=8.7 \mathrm{~Hz}$, 2H), 7.36-7.27 (m, 4H), $5.90(\mathrm{~s}, 1 \mathrm{H}), 2.04$ (brs, H). 71\% ee determined by HPLC with a Chiralcel OB- 
$\mathrm{H}$ column $(\mathrm{A} / \mathrm{B}=80: 20,0.8 \mathrm{~mL} / \mathrm{min}$, uv $230 \mathrm{~nm}): \mathrm{t}_{\mathrm{R}}=22.92 \mathrm{~min}\left(\right.$ minor), $\mathrm{t}_{\mathrm{R}}=25.07$ min (major). $[\alpha]_{\mathrm{D}}^{23}=-19.5(c=0.64, \mathrm{EtOH})$.

4-Chlorophenyl(3-bromophenyl)methanol (4b). ${ }^{1} \mathrm{H}-\mathrm{NMR}: \delta 7.53(\mathrm{~s}, 1 \mathrm{H}), 7.41(\mathrm{~d}, J=7.5 \mathrm{~Hz}, 1 \mathrm{H})$, 7.39-7.28 (m, 4H), 7.25-7.24 (m, 2H), $7.20(\mathrm{t}, J=7.5 \mathrm{~Hz}, 1 \mathrm{H}), 5.77(\mathrm{~s}, 1 \mathrm{H}), 2.04$ (brs, 2H). 55\% ee determined by HPLC with a Chiralcel OD-H column $(\mathrm{A} / \mathrm{B}=85: 15,0.8 \mathrm{~mL} / \mathrm{min}$, uv $230 \mathrm{~nm})$ : $\mathrm{t}_{\mathrm{R}}=7.80 \min$ (major), $\mathrm{t}_{\mathrm{R}}=8.58 \mathrm{~min}$ (minor). $[\alpha]_{\mathrm{D}}^{23}=+22.8(c=0.60, \mathrm{EtOH})$.

(S)-4-Methoxylphenyl(4-nitrophenyl)methanol (4c). ${ }^{1} \mathrm{H}-\mathrm{NMR}: \delta 8.19(\mathrm{~d}, J=8.7 \mathrm{~Hz}, 2 \mathrm{H}), 7.57$ (d, $J=8.4 \mathrm{~Hz}, 2 \mathrm{H}), 7.25(\mathrm{~d}, J=8.4 \mathrm{~Hz}, 2 \mathrm{H}), 6.89(\mathrm{~d}, J=8.7 \mathrm{~Hz}, 2 \mathrm{H}), 5.88(\mathrm{~s}, 1 \mathrm{H}), 3.80(\mathrm{~s}, 3 \mathrm{H}), 2.20$ (s, 1H). 54\%ee determined by HPLC with a Chiralpark AD-H column $(\mathrm{A} / \mathrm{B}=85: 15,0.8 \mathrm{~mL} / \mathrm{min}$, uv 254 $\mathrm{nm}): \mathrm{t}_{\mathrm{R}}=15.60 \min$ (minor), $\mathrm{t}_{\mathrm{R}}=19.25 \min$ (major). $[\alpha]_{\mathrm{D}}^{23}=+27.9(c=0.44, \mathrm{EtOH})$.

4-Methoxylphenyl(3-nitrophenyl)methanol (4d). ${ }^{1} \mathrm{H}-\mathrm{NMR}: \delta 8.28(\mathrm{~s}, 1 \mathrm{H}), 8.10(\mathrm{~d}, J=8.1 \mathrm{~Hz}, 1 \mathrm{H})$, $7.71(\mathrm{~d}, J=8.1 \mathrm{~Hz}, 1 \mathrm{H}), 7.49(\mathrm{t}, J=8.1 \mathrm{~Hz}, 1 \mathrm{H}), 7.27(\mathrm{~d}, J=6.6 \mathrm{~Hz}, 2 \mathrm{H}), 6.89(\mathrm{~d}, J=6.9 \mathrm{~Hz}, 2 \mathrm{H})$, $5.88(\mathrm{~s}, 1 \mathrm{H}), 3.80(\mathrm{~s}, 3 \mathrm{H}), 2.28(\mathrm{brs}, 1 \mathrm{H}) .24 \%$ ee determined by HPLC with a Chiralcel OD-H column $(\mathrm{A} / \mathrm{B}=85: 15,0.8 \mathrm{~mL} / \mathrm{min}$, uv $230 \mathrm{~nm}): \mathrm{t}_{\mathrm{R}}=15.08 \mathrm{~min}($ major $), \mathrm{t}_{\mathrm{R}}=16.23 \mathrm{~min}$ (minor). $[\alpha]_{\mathrm{D}}{ }^{23}=+23.8$ $(c=0.50, \mathrm{EtOH})$.

3, 5-Dimethylphenyl(phenyl)methanol (4e). ${ }^{1} \mathrm{H}-\mathrm{NMR}: \delta 7.42-7.28(\mathrm{~m}, 5 \mathrm{H}), 7.01(\mathrm{~s}, 2 \mathrm{H}), 6.93(\mathrm{~s}, 1 \mathrm{H})$, $5.77(\mathrm{~s}, 1 \mathrm{H}), 2.31(\mathrm{~s}, 6 \mathrm{H}), 2.18(\mathrm{brs}, 1 \mathrm{H}) .48 \%$ ee determined by HPLC with a Chiralcel OD-H column $(\mathrm{A} / \mathrm{B}=90: 10,0.8 \mathrm{~mL} / \mathrm{min}, \mathrm{uv} 254 \mathrm{~nm}): \mathrm{t}_{\mathrm{R}}=8.67 \mathrm{~min}($ minor $), \mathrm{t}_{\mathrm{R}}=9.58 \mathrm{~min}($ major $) .[\alpha]_{\mathrm{D}}{ }^{23}=+20.4(c$ $=0.65, \mathrm{EtOH})$.

\section{Conclusions}

In summary, we haved reported the asymmetric aryl transfer to aldehydes with arylboronic acids as aryl sources in the presence of the chiral 2,2'-bispyrrolidine-based ligand L6. The corresponding diarylmethanols could be obtained in high yields with moderate to good enantioselectivities. Further work on the asymmetric addition mechanism and the broad application of chiral 2,2'-bispyrrolidinebased ligands in other asymmetric catalytic reactions are now in progress in our laboratory.

\section{Acknowledgements}

We gratefully acknowledge the National Natural Science Foundation of China (20832001, 20972065, 21074054) and the National Basic Research Program of China (2007CB925103, 2010CB923303) for their financial support. The Major Scientific and Technological Special Project (2009ZX09103-081) is also acknowledged.

\section{References and Notes}

1. Shafi'ee, A.; Hite, G. Absolute configurations of the pheniramines, methyl phenidates, and pipradrols. J. Med. Chem. 1969, 12, 266-270. 
2. Ebnǒther, A.; Weber, H.P. Synthesis and absolute configuration of clemastine and its isomers. Helv. Chim. Acta 1976, 59, 2462-2468.

3. Meguro, K.; Aizawa, M.; Sohda, T.; Kawamatsu, A.; Nagaoka, A. New 1,4-dihydropyridine derivatives with potent and long-lasting hypotensive effect. Chem. Pharm. Bull. 1985, 33, 3787-3797.

4. Toda, F.; Tanaka, K.; Koshiro, K. A new preparative method for optically active diarylcarbinols. Tetrahedron Asymmetry 1991, 2, 873-874.

5. Casy, A.F.; Drake, A.F.; Ganellin, C.R.; Mercer, A.D.; Upton, C. Stereochemical studies of chiral H-1 antagonists of histamine: The resolution, chiral analysis, and biological evaluation of four antipodal pairs. Chirality 1992, 4, 356-366.

6. Stanev, S.; Rakovska, R.; Berova, N.; Snatzke, G. Synthesis, absolute configuration and circular dichroism of some diarylmethane derivatives. Tetrahedron Asymmetry 1995, 6, 183-198.

7. Botta, M.; Summa, V.; Corelli, F.; Di Pietro, G.; Lombardi, P. Synthesis of aryl 2-benzofuranyl and 2-indolyl carbinols of high enantiomeric purity via palladium-catalyzed heteroannulation of chiral arylpropargylic alcohols. Tetrahedron Asymmetry 1996, 7, 1263-1266.

8. Torrens, A.; Castrillo, J.A.; Claparols, A.; Redondo, J. Enantioselective synthesis of (R)- and (S)cizolirtine. Application of oxazaborolidine-catalyzed asymmetric borane reduction to azolyl phenyl ketones. Synlett 1999, 765-767.

9. Corey, E.J.; Helal, C.J. Reduction of carbonyl compounds with chiral oxazaborolidine catalysts: A new paradigm for enantioselective catalysis and a powerful new synthetic method. Angew. Chem. Int. Ed. 1998, 37, 1986-2012.

10. Corey, E.J.; Bakshi, R.K.; Shibata, S. Highly enantioselective borane reduction of ketones catalyzed by chiral oxazaborolidines. Mechanism and synthetic implications. J. Am. Chem. Soc. 1987, 109, 5551-5553.

11. Corey, E.J. New enantioselective routes to biologically interesting compounds. Pure Appl. Chem. 1990, 62, 1209-1216.

12. Ohkuma, T.; Koizumi, M.; Ikehira, H.; Yokozawa, T.; Noyori, R. Selective hydrogenation of benzophenones to benzhydrols. Asymmetric synthesis of unsymmetrical diarylmethanols. Org. Lett. 2000, 2, 659-662.

13. Noyori, R.; Ohkuma, T. Rapid, productive and stereoselective hydrogenation of ketones. Pure Appl. Chem. 1999, 71, 1493-1501.

14. $\mathrm{Pu}, \mathrm{L} . ; \mathrm{Yu}, \mathrm{H} .-\mathrm{B}$. Catalytic asymmetric organozinc additions to carbonyl compounds. Chem. Rev. 2001, 101, 757-824.

15. Bolm, C.; Hildebrand, J.P.; Muńiz, K.; Hermanns, N. Catalyzed asymmetric arylation reactions. Angew. Chem. Int. Ed. 2001, 40, 3284-3308.

16. Schmidt, F.; Stemmler, R.T.; Rudolph, J.; Bolm, C. Catalytic asymmetric approaches towards enantiomerically enriched diarylmethanols and diarylmethylamines. Chem. Soc. Rev. 2006, 35, 454-470.

17. Langer, F.; Schwink, L.; Devasagayaraj, A.; Chavant, P.-Y.; Knochel, P. Preparation of functionalized dialkylzincs via a boron-zinc exchange. Reactivity and catalytic asymmetric addition to aldehydes. J. Org. Chem. 1996, 61, 8229-8243. 
18. Oppolzer, W.; Radinov, R.N.; Sayed, E.E. Catalytic asymmetric synthesis of macrocyclic (E)allylic alcohols from $\omega$-alkynals via intramolecular 1 -alkenylzinc/aldehyde additions. J. Org. Chem. 2001, 66, 4766-4770.

19. Dahmen, S.; Brase, S. [2, 2] Paracyclophane-based N,O-ligands in alkenylzinc additions to Aldehydes. Org. Lett. 2001, 3, 4119-4122.

20. Bolm, C.; Rudolph, J. Catalyzed asymmetric aryl transfer reactions to aldehydes with boronic acids as aryl source. J. Am. Chem. Soc. 2002, 124, 14850-14851.

21. Rudolph, J.; Schmidt, F.; Bolm, C. Highly enantioselective synthesis of secondary alcohols using triphenylborane. Adv. Synth. Catal. 2004, 346, 867-872.

22. Braga, A.L.; Luedtke, D.S.; Vargas, F.; Paixao, M.W. Catalytic enantioselective arylation of aldehydes: Boronic acids as a suitable source of transferable aryl groups. Chem. Commun. 2005, 2512-2514.

23. Wu, X.-Y.; Liu, X.-Y.; Zhao, G. Catalyzed asymmetric aryl transfer reactions to aldehydes with boroxines as aryl source. Tetrahedron Asymmetry 2005, 16, 2299-2305.

24. Liu, X.-Y.; Wu, X.-Y.; Chai, Z.; Wu, Y.-Y.; Zhao, G.; Zhu, S.-Z. Highly effective and recyclable dendritic ligands for the enantioselective aryl transfer reactions to aldehydes. J. Org. Chem. 2005, 70, 7432-7435.

25. Bolm, C.; Schmidt, F.; Zani, L. Synthesis of new chiral hydroxy oxazolines and their use in the catalytic asymmetric phenyl transfer to aldehydes. Tetrahedron Asymmetry 2005, 16, 1367-1376.

26. Dahmen, S.; Lormann, M. Triarylborane ammonia complexes as ideal precursors for arylzinc reagents in asymmetric catalysis. Org. Lett. 2005, 7, 4597-4600.

27. Jia, X.-F.; Fang, L.; Lin, A.-J.; Pan, Y.; Zhu, C.-J. Highly efficient and facile aryl transfer to aldehydes using $\mathrm{ArB}(\mathrm{OH})_{2}-\mathrm{GaMe}_{3}$. Synlett 2009, 3, 495-499.

28. Ji, J.-X.; Wu, J.; Au-Yeung, T.-T.-L.; Yip, C.W.; Haynes, R.K.; Chan, A.S.C. Highly enantioselective phenyl transfer to aryl aldehydes catalyzed by easily accessible chiral tertiary aminonaphthol. J. Org. Chem. 2005, 70, 1093-1095.

29. Ito, K.; Tomita, Y.; Katsuki, T. Enantioselective phenyl transfer to aldehydes using 1,1'-bi-2naphthol-3,3'-dicarboxamide as chiral auxiliary. Tetrahedron Lett. 2005, 46, 6083-6086.

30. Braga, A.L.; Milani, P.; Vargas, F.; Paixāo, M.W.; Sehnem, J.A. Modular chiral thiazolidine catalysts in asymmetric aryl transfer reactions. Tetrahedron Asymmetry 2006, 17, 2793-2797.

31. Wu, P.-Y.; Wu, H.-L.; Uang, B.-J. Asymmetric synthesis of functionalized diarylmethanols catalyzed by a new $\gamma$-amino thiol. J. Org. Chem. 2006, 71, 833-835.

32. Lu, G.; Kwong, F.-Y.; Ruan, J.W.; Li, Y.-M.; Chan, A.S.C. Highly enantioselective addition of in situ prepared arylzinc to aldehydes catalyzed by a series of atropisomeric binaphthyl-derived amino alcohols. Chem. Eur. J. 2006, 12, 4115-4120.

33. Jin, M.J.; Sarkar, S.M.; Lee, D.H.; Qiu, H.L. Highly enantioselective aryl transfer to aldehydes: A remarkable effect of sulfur substitution in amino thioacetate ligands. Org. Lett. 2008, 10, 1235-1237.

34. Huang, X.-B.; Wu, L.-L.; Xu, J.-Q.; Zong, L.-L.; Hu, H.-W.; Cheng, Y.-X. Enantioselective arylation of aldehydes catalyzed by a soluble optically active polybinaphthols ligand. Tetrahedron Lett. 2008, 49, 6823-6826. 
35. DeBerardinis, A.M.; Turlington, M.; Pu, L. Activation of functional arylzincs prepared from aryl iodides and highly enantioselective addition to aldehydes. Org. Lett. 2008, 10, 2709-2712.

36. Wang, M.-C.; Wang, X.-D.; Ding, X.; Liu, Z.-K. Catalytic asymmetric aryl transfer: Highly enantioselective preparation of (R)- and (S)-diarylmethanols catalyzed by the same chiral ferrocenyl aziridino alcohol. Tetrahedron 2008, 64, 2559-2564.

37. Ruan, J.-W.; Lu, G.; Xu, L.-J.; Li, Y.-M.; Chan, A.S.C. Catalytic asymmetric alkynylation and arylation of aldehydes by an $\mathrm{H}_{8}$-binaphthyl-based amino alcohol ligand. Adv. Synth. Catal. 2008, 350, 76-84.

38. Wang, M.-C.; Zhang, Q.-J.; Zhao, W.-X.; Wang, X.-D.; Ding, X.; Jing, T.-T.; Song, M.-P. N(Ferrocenylmethyl)azetidin-2-yl(diphenyl)methanol for catalytic asymmetric addition of organozinc reagents to aldehydes. J. Org. Chem. 2008, 73, 168-176.

39. Braga, A.L.; Paixa, M.W.; Westermann, B.; Schneider, P.H.; Wessjohann, L.A. Acceleration of arylzinc formation and its enantioselective addition to aldehydes by microwave irradiation and aziridine-2-methanol catalysts. J. Org. Chem. 2008, 73, 2879-2882.

40. Salvi, L.; Kim, J.G.; Walsh, P.J. Practical catalytic asymmetric synthesis of diaryl-, aryl heteroaryl-, and diheteroarylmethanols. J. Am. Chem. Soc. 2009, 131, 12483-12493.

41. Wouters, A.D.; Trossini, G.H.G.; Stefani, H.A.; Lüdtke, D.S. Enantioselective arylations catalyzed by carbohydrate-based chiral amino alcohols. Eur. J. Org. Chem. 2010, 2351-2356.

42. Alexakis, A.; Mangeney, P. Advanced Asymmetric Synthesis; Stephenson, G.R., Ed.; Chapman \& Hall: London, UK, 1996; Chapter 5, p. 93.

43. Bennani, Y.L.; Hannessian, S. Trans-1,2-diaminocyclohexane derivatives as chiral reagents, scaffolds, and ligands for catalysis: Applications in asymmetric synthesis and molecular recognition. Chem. Rev. 1997, 97, 3161-3195.

44. Lucet, D.; Le Gall, T.; Mioskowski, C. The chemistry of vicinal diamines. Angew. Chem. Int. Ed. 1998, 37, 2580-2627.

45. Hirama, M.; Oishi, T.; Ito, S. Asymmetric dihydroxylation of alkenes with osmium tetroxide: Chiral N, N'-dialkyl-2,2'-bipyrrolidine complex. Chem. Commun. 1989, 10, 665-666.

46. Oishi, T; Hirama, M. Highly enantioselective dihydroxylation of trans-disubstituted and monosubstituted olefins. J. Org. Chem. 1989, 54, 5834-5835.

47. Oishi, T.; Hirama, M.; Sita, L.R.; Masamune, S. Synthesis of chiral 2, 2'-bipyrrolidine derivatives. Synthesis 1991, 789-792.

48. Kotsuki, H.; Kuzume, H.; Gohda, T.; Fukuhara, M.; Ochi, M.; Oishi, T.; Hirama, M.; Shirot, M. New convenient, enantiospecific synthesis of $(\mathrm{S}, \mathrm{S})$ - and $(\mathrm{R}, \mathrm{R})-2,2$ '-bipyrrolidine derivatives. Tetrahedron Asymmetry 1995, 6, 2227-2236.

49. Alexakis, A.; Tomassini, A.; Chouillet, C.; Roland, S.; Mangeney, P.; Bernardinelli, G. A new efficient synthesis of (R, R)-2,2'-bipyrrolidine: An interesting chiral 1,2-diamine with $C_{2}$ symmetry. Angew. Chem. Int. Ed. 2000, 39, 4093-4095.

50. Denmark, S.E.; Fu, J.-P.; Lawler, M.J. (R, R)-2,2'- bipyrrolidine and (S, S)-2,2'-bipyrrolidine: Useful ligands for asymmetric synthesis. Org. Synth. 2006, 83, 121-130.

51. Denmark, S.E.; Fu, J.-P. Catalytic, Enantioselective addition of substituted allylic trichlorosilanes using a rationally-designed 2,2'-bispyrrolidine-based bisphosphoramide. J. Am. Chem. Soc. 2001, 123, 9488-9489. 
52. Alexakis, A.; Andrey, O. Diamine-catalyzed asymmetric michael additions of aldehydes and ketones to nitrostyrene. Org. Lett. 2002, 4, 3611-3614.

53. Andrey, O.; Alexakis, A.; Bernardinelli, G. Asymmetric michael addition of $\alpha$-Hydroxyketones to nitroolefins catalyzed by chiral diamine. Org. Lett. 2003, 5, 2559-2561.

54. Andrey, O.; Alexakis, A.; Tomassini, A.; Bernardinelli, G. The use of N-alkyl-2,2'-bipyrrolidine derivatives as organocatalysts for the asymmetric michael addition of ketones and aldehydes to nitroolefins. Adv. Synth. Catal. 2004, 346, 1147-1168.

55. Mossé, S.; Alexakis, A. First organocatalyzed asymmetric michael addition of aldehydes to vinyl Sulfones. Org. Lett. 2005, 7, 4361-4364.

56. Alexakis, A.; Tomassini, A.; Andrey, O.; Bernardinelli, G. Diastereoselective alkylation of (arene)tricarbonylchromium and ferrocene complexes using a chiral, $\mathrm{C}_{2}$-symmetrical 1,2-diamine as auxiliary. Eur. J. Org. Chem. 2005, 1332-1339.

57. Denmark, S.E.; Fu, J.-P.; Lawler, M.J. Chiral phosphoramide-catalyzed enantioselective addition of allylic trichlorosilanes to aldehydes. Preparative studies with bidentate phosphorus-based amides. J. Org. Chem. 2006, 71, 1523-1536.

58. Chen, M.S.; White M.C. A predictably selective aliphatic C-H oxidation reaction for complex molecule synthesis. Science 2007, 318, 783-787.

59. Suzuki, K.; Oldenburg, P.D.; Que, L., Jr. Iron-catalyzed asymmetric olefin cis-dihydroxylation with 97\% enantiomeric excess. Angew. Chem. Int. Ed. 2008, 47, 1887-1889.

60. Sergeeva, E.; Kopilov, J.; Goldberg, I.; Kol, M. Salan ligands assembled around chiral bipyrrolidine: Predetermination of chirality around octahedral Ti and $\mathrm{Zr}$ centres. Chem. Commun. 2009, 21, 3053-3055.

61. Sergeeva, E.; Kopilov, J.; Goldberg, I.; Kol, M. 2,2'-Bipyrrolidine versus 1,2-diaminocyclohexane as chiral cores for helically wrapping diamine-diolate ligands. Inorg. Chem. 2009, 48, 8075-8077.

62. Jia, Y.-X.; Zhu, S.-F.; Yang, Y.; Zhou Q.-L. Asymmetric friedel-crafts alkylations of indoles with nitroalkenes catalyzed by Zn(II)-bisoxazoline complexes. J. Org. Chem. 2006, 71, 75-80.

63. Arai, T.; Yokoyama, N. Tandem Catalytic Asymmetric friedel-crafts/henry reaction: Control of three contiguous acyclic stereocenters. Angew. Chem. Int. Ed. 2008, 47, 4989-4992.

Sample Availability: Samples of the compounds are available from the authors.

(C) 2011 by the authors; licensee MDPI, Basel, Switzerland. This article is an open access article distributed under the terms and conditions of the Creative Commons Attribution license (http://creativecommons.org/licenses/by/3.0/). 